\title{
Ulcer in a dialysis patient: calciphylaxis or something simpler
}

\author{
Amr Mohamed ${ }^{1}$ and Salem Thabet $^{1}$ \\ ${ }^{1}$ Rochester General Hospital
}

June 12, 2021

\begin{abstract}
In our everyday practice, we see ulcers in dialysis patients; we usually think blindly about calciphylaxis. Sometimes even we start patients on empiric treatment based on that assumption, but let us assume that something simpler may be going on, as seen in this case.
\end{abstract}

Title : Ulcer in a dialysis patient: calciphylaxis or something simpler

Author : Mohamed Amr, MD.

Salem Thabet, MD.

Affiliation : Department of Internal Medicine, Rochester General Hospital, Rochester, NY.

Corresponding Author: Mohamed Amr, MD

Contact number : 718-764-7202

Affiliation Address : 1425 Portland Avenue, Rochester, NY, 14621.

Email address : amrelwagdycardiol@gmail.com

Funding source : none

Conflict of interest : none

Article type : Clinical image

Word count : 230

Consent: patient verbal permission had been obtained to publish his images. The approval had been documented in his electronic medical record. In the setting of the COVID pandemic, the use of paperwork is prohibited per Rochester regional health policy

A 56-year-old male with a past medical history of end-stage renal disease on regular hemodialysis, streptococcal endocarditis four years ago, COVID-19 pneumonia three months ago, Who presented to the ED with a complaint of wounds on both legs, as shown in the image.

He reported severe itching for two months, and the ulcers started three weeks before presentation. The ulcers were initially painful, later painless, initially on the right foot, later on, both right and left foot, and the lower legs on both sides. He denied IV drug abuse, and his drug screen had been negative. His physical exam had been normal other than the leg ulcers, with no systemic signs of infection and no fevers.

Infective endocarditis had been ruled out with negative blood cultures and a negative transthoracic echocardiogram. Given that the clinical picture was not clear, multiple skin biopsies were performed, and it showed 
no evidence of vasculitis, or infectious process, or calciphylaxis. The epidermis adjacent to the ulcer is reactive/hyperplastic, and the changes were suggested to be secondary to CKD-associated pruritis. The patient received Triamcinolone cream with Antihistaminic and local wound care, and the ulcers improved.

The key clinical message To know the differential diagnosis of ulcers in a dialysis patient. We thought about vasculitis, infectious process, and calciphylaxis, but it ended up being something much more straightforward related to CKD pruritis.

\section{Figure legend}

Figure 1: shows bilateral lower extremity ulcers in geographic distribution on both lower extremities.
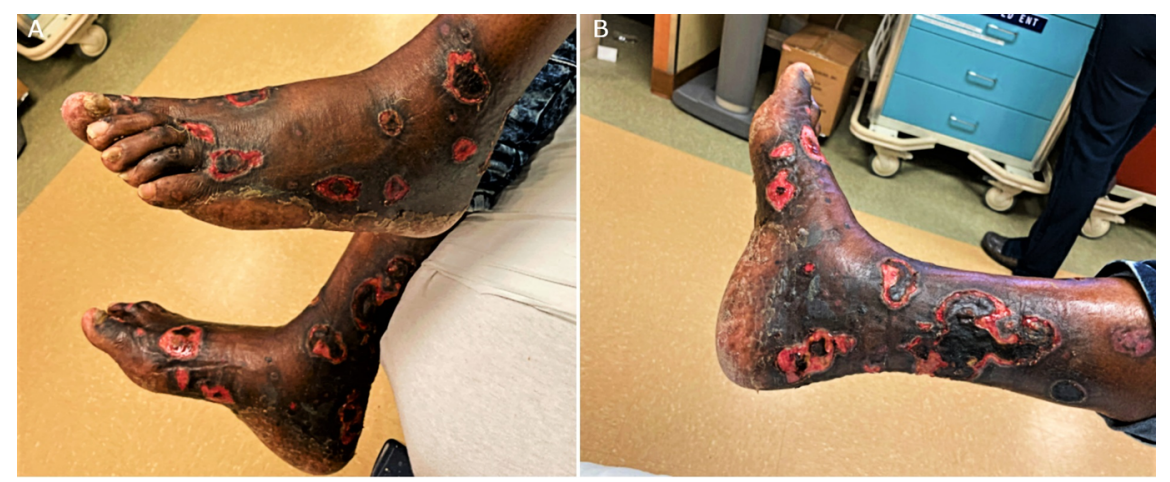